\title{
The Large Analog Bandwidth Recorder and Digitizer with Ordered Readout (LABRADOR) ASIC
}

\author{
G.S. Varner ${ }^{\mathrm{a}, *}$ L.L. Ruckman ${ }^{\mathrm{a}}$, J.W. Nam ${ }^{\mathrm{b}}$, R.J. Nichol ${ }^{\mathrm{c}}$ J. Cao $^{\mathrm{d}}$, P.W. Gorham ${ }^{\mathrm{a}}$ and \\ M. Wilcox ${ }^{\mathrm{e}}$ \\ ${ }^{a}$ Department of Physics and Astronomy, University of Hawaii, 2505 Correa Road, Honolulu HI 96822, USA \\ ${ }^{\mathrm{b}}$ Department of Physics and Astronomy, University of California at Irvine, Frederick Reines Hall, Irvine CA 92697, USA \\ ${ }^{\mathrm{c}}$ Department of Physics and Astronomy, University College London, London WC1E 6BT, UK \\ ${ }^{\mathrm{d}}$ was at the Univ. of Hawaii, now with NeuroPace Inc., 1375 Shorebird Way, Mountain View CA 94043, USA \\ ${ }^{\mathrm{e}}$ was at the Univ. of Hawaii, now with Oceanit Laboratories Inc., 9565 Kaumualii Hwy., Waimea HI 96769, USA
}

\begin{abstract}
Three generations of full-custom analog integrated circuits designed for low-power, high-speed sampling of RadioFrequency (RF) transients in excess of the Nyquist minimum have been developed. These $0.25 \mu m$ CMOS devices are denoted the Large Analog Bandwidth Recorder and Digitizer with Ordered Readout (LABRADOR) ASICs and finally consist of 9 channels of 260 deep sampling. Continuous sampling is provided with common stop capability. Input analog bandwidth is approximately $1 \mathrm{GHz}$ and sampling speeds are adjustable from 0.02 to $3.7 \mathrm{GSa} / \mathrm{s}$. Completely parallel internal conversion supports 12-bit digitization and readout of all 2340 cells in under $50 \mu s$.
\end{abstract}

\section{Introduction}

Observation of the early universe through neutrino messengers of the highest possible energies requires a detector of enormous instrumented volume. One promising means to observe such a large, radio-transparent target is viewing the Antarctic ice shelf via high altitude balloon [1]. Such a balloon-borne detector needs hundreds of highspeed sampling channels (multi-event buffering), operating over a frequency band from 200-1200 $\mathrm{MHz}$ [2]. Since all power must come from solar panels, and heat dissipation is a major problem, commercial flash ADCs were precluded.

\footnotetext{
* Corresponding author. Tel./fax: +001 808-956-2987. Email address: varner@phys.hawaii.edu (G.S. Varner).
}

For at least two decades a number of Switched Capacitor Array (SCA) devices have been reported in the high energy physics literature, for example $[3,4,5]$, and many with sampling speeds high enough for greater than Nyquist sampling of a $\mathrm{GHz}$ analog bandwidth signal. These GSa/s devices have been used for low and high energy neutrino detection [6], particle physics $[7,8]$ and gamma-ray astronomy [9]. However, despite such high sampling speeds, all of these devices have analog bandwidth cutoffs which limit their use at UHF frequencies and above.

We present here the results of three generations of a high analog bandwidth ASIC designed to meet these instrumentation needs. 


\section{Architecture}

A number of different CMOS SCA architectures have been discussed in the literature. An excellent summary of the storage circuit details and performance may be found in Ref. [10]. As will be seen below, in order to couple in high analog bandwidth it is necessary to limit the parasitic and storage capacitance of the SCA array. Thus a compact, minimal storage array was considered and initial prototyping looked promising [11]. This choice of a compact storage matrix was guided and synergistic with very similar storage architectures being explored for Monolithic Active Pixel Sensors (MAPS) [12] for charged particle tracking.

\subsection{Theory of Operation}

Employment of SCA techniques in CMOS processes have been effective in the areas of basic signal processing, continuous filter design, and programmable capacitor arrays, used for Digital-toAnalog (DAC) and Analog-to-Digital (ADC) conversion. As elements of a basic programmable filter, a simple inline capacitor between two switches may be used to form a frequency-controlled resistor, with resistance $R$ given by [13]:

$R=\frac{1}{f_{c} C}$

for a given capacitor $C$, being switched at frequency $f_{c}[\mathrm{~Hz}]$. Almost arbitrarily complex filters, composed of these variable $R$ and $C$ configurations, can be formed and expressed in terms of poles and zeros in a transfer function, the mathematics of which is conveniently described via the $z$-Transform [14], a staple of modern signal processing. As an example, from these simple building blocks, first order filters can be constructed as represented by the transfer function:

$H(z)=K \frac{b_{0}+b_{1} z^{-1}}{1+a_{1} z^{-1}}$

where $z^{-1}=e^{-i \omega T}, T=1 / f_{c},-1<a_{1}<1$, and $K$ is an overall normalization constant. Through choice of constants, one can form a Low-pass filter $\left(b_{0}-b_{1}=0\right)$ :
$H(z)=K \frac{1+z^{-1}}{1+a_{1} z^{-1}}$

or a High-pass filter $\left(b_{0}=-b_{1}\right)$ :

$H(z)=K \frac{1-z^{-1}}{1+a_{1} z^{-1}}$

Since first-order filters only have one real pole, they cannot directly realize band-pass or notch filters. More flexible and universal are filters of secondorder and beyond. Second order SC filters are often called biquad circuits and have may be expressed as

$H(z)=K \frac{b_{0}+b_{1} z^{-1}+b_{2} z^{-2}}{1+a_{1} z^{-1}+a_{2} z^{-2}}$

and is analogous to the continous time case where the transfer function may be represented by

$H(s)=K_{0} \frac{s^{2}+d_{1} s+d_{0}}{s^{2}+c_{1} s+c_{0}}$

where as long as the sampling frequency is much higher than the signals of interest, the approximation $z^{-1} \simeq 1-i \omega T$ may be used. And from this point, standard pole-zero analysis can be used.

Beyond simple synthesis of rather complex filters using standard tools, the true power of this technique lies in pairing such SCA processing with operational amplifiers on an integrated circuit to achieve powerful sampling and signal manipulation capabilities. For instance, in analogy with an R-2R ladder topology, a multiplying DAC may be expressed using an array of switches and capacitors with the simple transfer function [15]

$H(z)=z^{-\frac{1}{2}} \sum_{i=1}^{n} 2^{-i} b_{i}$

and ignoring the half-period delay indicated by the $z^{-\frac{1}{2}}$, can synethize an output voltage $v_{\text {out }}$ based upon a reference voltage $v_{r e f}$ via the expression

$v_{\text {out }}=v_{\text {ref }} \sum_{i=1}^{n} \frac{b_{i}}{2^{i}}$

with the $b_{i}$ being the binary-coded digital signal, precisely as expected for a DAC. ADC topologies are now myriad and the focus of this paper is on a specific type - the transient waveform recorder. 
In some ways this makes use of the simplest SCA structure of them all, the Sample-and-Hold $(\mathrm{S} / \mathrm{H})$ circuit. The great power of the papers referenced above derives from the ever increasing speed and compactness of deep submicron CMOS processes.

While an idealized waveform recorder is simply an array of $\mathrm{S} / \mathrm{H}$ circuits, parasitic capacitances require consideration of parasitic circuits like those referenced above. For the specific application at hand, parasitic inductances and capacitances are critical to storing analog waveforms with frequency content in the Giga-Hertz range.

\subsection{Bandwidth Limitations}

In order for an SCA storage device to be useful, it must have a decent number of storage cells. Load capacitance increases as a function of the number of switches connected to the incoming signal line, as well as the resistance-shielded storage capacitances when the switches are closed. For a properly coupled $50 \Omega$ stripline into an ASIC, for a purely capacitive storage array, the $3 \mathrm{~dB}$ roll-off is given as

$f_{3 \mathrm{~dB}}=\frac{1}{2 \pi Z_{0} C}$.

Therefore, to obtain a $3 \mathrm{~dB}$ bandwidth of $1.2 \mathrm{GHz}$, the pure capacitance must be limited to approximately $2.65 p F$. This value is already smaller than that of the high-ESD protection diodes $(\sim 10 p F)$ provided in a standard design library used and therefore the input protection must be modified. A more accurate assessment of the input coupling performance requires a refined description of the input circuit model and will be discussed in much more architecture-specific detail below. In summary, to realize $1 \mathrm{GHz}$ of analog bandwidth with good coupling, will use the following design principles:

(i) $50 \Omega$ stripline everywhere

(ii) minimize input protection capacitance

(iii) minimize switch drain, storage capacitance

Based upon these considerations, efforts have been made to maintain a $50 \Omega$ coupling across the sampling array inside the ASIC. As a trade-off between storage depth and parasitic drain capaci- tance, 256 samples per input was chosen. Finally, the size of the storage capacitance was studied.

\subsection{Storage Limitations}

Limits are imposed on the minimum size possible for the storage capacitor. Since for a S/H circuit there is no means to perform a Correlated Double Sampling, an ambiguity in the actual stored value is given in terms of electron counting statistics by the usual expression

$v_{r m s}=\sqrt{\frac{k T}{C}}$

where $k$ is Boltzmann's constant and temperure $T$ is in Kelvin. Matching the 12-bits of dynamic range of the sample storage conversion to a Wilkinson ramp voltage range of about $1 \mathrm{~V}$, a slope of approximately $0.25 \mathrm{mV} /$ least count is realized. At this level of sensitivity, the impact of the choice of storage capacitance value is seen in Fig. 1. At the upper left of this figure is a schematic representation of the basic storage cell for the first two generations of ASIC that utilized a transimpedance storage architecture. A reference value of $78 f F$ is shown - about matching the least count of the ADC as shown.

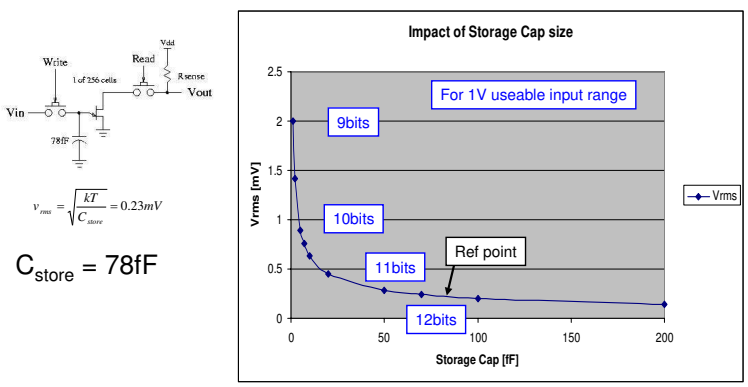

Fig. 1. Noise limited sampling resolution as a function of storage capacitance value.

In the last generation a different readout is employed, although the same basic NMOS transistor gate capacitance storage is used. This constraint on minimum size is subsequently considered in the choice of storage capacitance. However reducing the storage capacitance too much makes switch charge injection and leakage current effects more prominent. 


\subsection{Architectural Details}

Three generations of LABRADOR architecture ASIC have been designed, fabricated and tested. Their key features are summarized in Table 1. All have been fabricated in the TSMC $0.25 \mu \mathrm{m}$ CMOS (LO) process and have been packaged in a 100pin plastic TQFP package. Economics and package performance simulations [11] drove this decision. BGA packages were considered and may be used in the future to reduce the contribution due to lead inductance, however all test results are shown for this same $16.6 \times 16.6 \mathrm{~mm}$ plastic package.

Table 1

Summary of three LABRADOR generations, where for brevity they will be referred to by a shortened designation; e.g. $L A B R A D O R 1=L A B 1$.

\begin{tabular}{|l|c|c|c|}
\hline Item & LAB1 & LAB2 & LAB3 \\
\hline \hline \# of RF inputs & 8 & 8 & 9 \\
\hline Samples/input & 256 & 256 & 260 \\
\hline Total samples & 2048 & 2048 & 2340 \\
\hline$\#$ of ADCs & 128 & 128 & 2340 \\
\hline ADC Conversion cycles & 16 & 16 & 1 \\
\hline Readout latency $[\mu \mathrm{s}]$ & 2200 & 2200 & $\leq 50$ \\
\hline Analog MUX out $[\mu \mathrm{s}]$ & 25.6 & 25.6 & N/A \\
\hline DC GND ref. & no & yes & no \\
\hline Analog out & yes & yes & no \\
\hline $50 \Omega$ term. & end & end & input \\
\hline \hline
\end{tabular}

In contrast to the first two generations of LABRADOR ASIC, the third generation was a purely digital output device, changed input termination scheme to be at the input, and went to a massive array of Wilkinson ADCs (one per pixel). These differences and lessons learned will be highlighted below. The architecture of first two ASICs is illustrated schematically in Fig. 2. Examining Table 1, the primary difference between LAB1 and LAB2 was the attempt to provide a means to internally bias the RF inputs. This circuit did not work well due to high resistance noise coupling. LAB1 results are similar, though better in all cases. Eight RF input channels are each sampled by an array of 256 SCA storage cells. Sampling occurs continually until a trigger signal is generated.

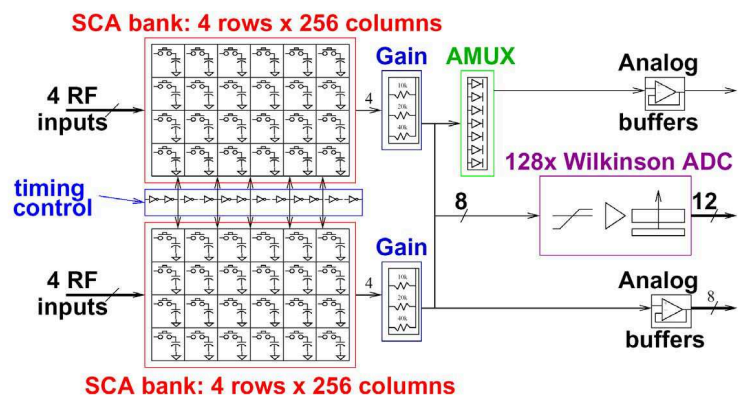

Fig. 2. Block diagram of the LAB1/LAB2 architecture. Samples are stored for the $8 \mathrm{RF}$ inputs in an array of 256 storage cells. Writing is controlled by a write pointer that continuously cycles across the array and stored values are held upon receipt of trigger signal. Stored values are then addressed (gain adjusted) and either stored for conversion in an array of 128 Wilkinson ADCs or multiplexed off-chip for external conversion.

At this point the analog samples are held and not overwritten. These stored values are then selected and a transimpedance relay of the stored charge is made, which is either stored into input samples of an array of 128 channels of Wilkinson ADC or analog multiplexed and transferred off-chip for external ADC conversion. A die photograph of the approximately $10 \mathrm{~mm}^{2}$ LAB1 device is shown in Fig. 3.

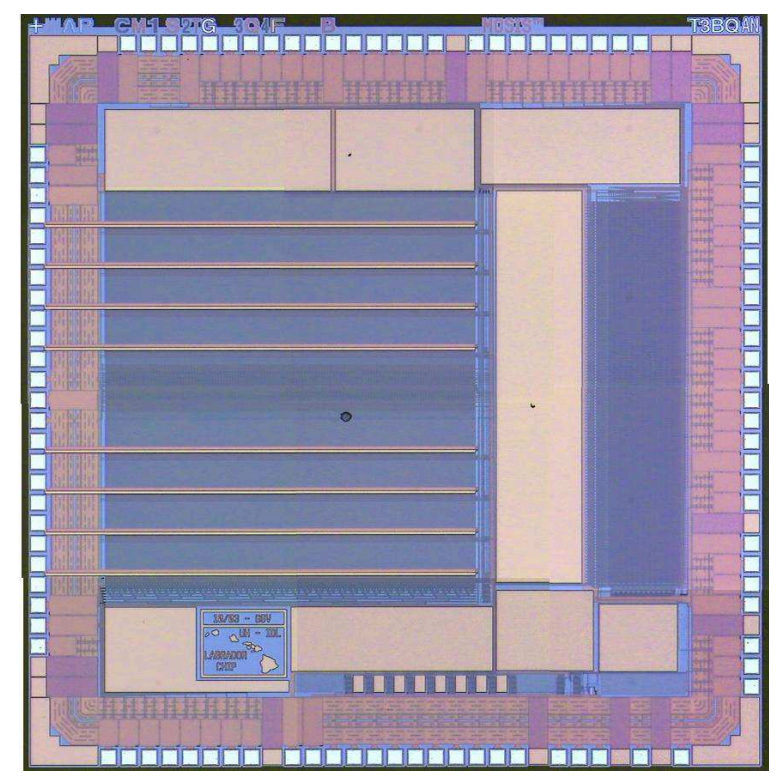

Fig. 3. A die photograph of the LABRADOR1 ASIC. 


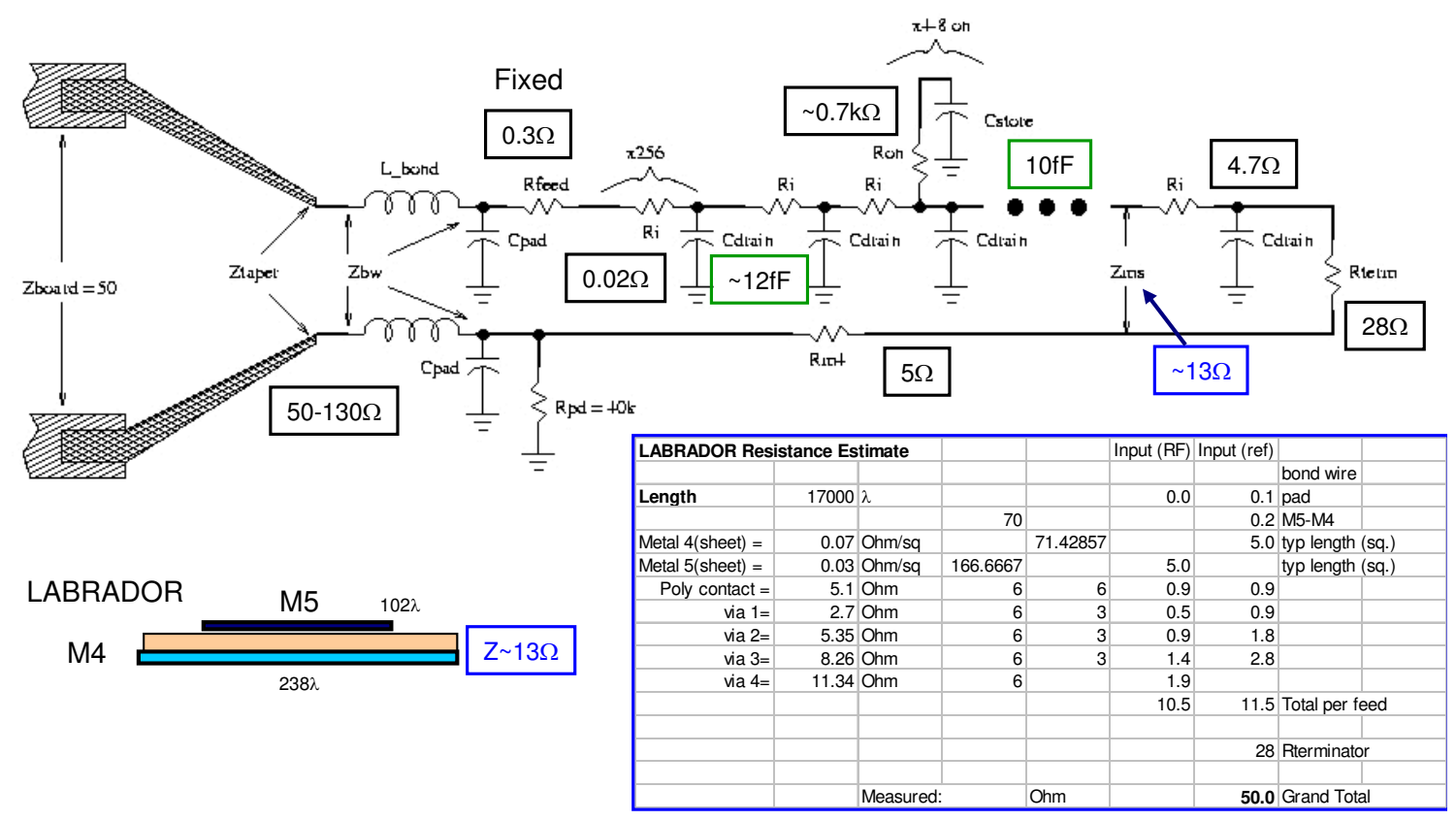

Fig. 4. Schematic representation and resistance breakdown of the LAB1 signal chain. Effects due to both resistive drop across the sampling array, as well as low impedance of the on-chip stripline, were observed in testing.

Layout of the LAB1 ASIC quite directly follows the arrangement of the functional blocks in the schematic diagram. While efforts were made to optimize the coupling of the input signal based on earlier efforts with the STRAW [11] architecture, the choice of LAB1 input structure represented a compromise, as shown in Fig. 4. Signals are straight input shots on the left and terminated in a $28 \Omega$ resistor at the right. This choice is a trade-off between widening the signal trace, which would lower the microstrip impedance even below the $Z_{0}=13 \Omega$ shown, or having even larger resistive losses across the array. These resistive losses made for a vexing amplitude-dependence across the array. To address this issue in LAB3, a $50 \Omega$ termination resistor is placed directly at the input to the detector. The termination resistor was removed from the array end. Since offset biasing could be performed directly at this input termination, the resistance of the signal line was unimportant and the on-chip stripline could be made exactly $Z=50 \Omega$. Any reflection at the end of the array would be backterminated, though this stub is short. At maximum signal frequency of $1.2 \mathrm{GHz}$, for a stripline of $2 \mathrm{~mm}$ long (about 10 ps at $v_{\text {prop }} \simeq \frac{2}{3} c$ ), the phase introduced by this stub is about

$2 \cdot \frac{10 \mathrm{ps}}{(1.2 \mathrm{GHz})^{-1}} \cdot\left(360^{\circ}\right) \simeq 8.6^{\circ}$

which is acceptable, though for operation at higher frequencies, such effects may be non-negligible. In all cases the input protection diodes have been completely removed. Current discharge is provided through a $20 k \Omega$ pull-down resistor to ground and voltage clamping is provided by external back-toback RF diodes.

Other lessons gleaned from the first two LABRADOR generations included observing that while having analog samples available for external digitization has merits, non-linearities in the transimpedance response and temperature dependence were major issues. As space was available to permit completely parallel conversion of all 9 channels by 260 samples, in-situ conversion was adopted, as illustrated in Fig. 5. Including four extra "tail" samples avoids a sampling record gap during the interval in which the write pointer is returning to the beginning of the sampling window. 


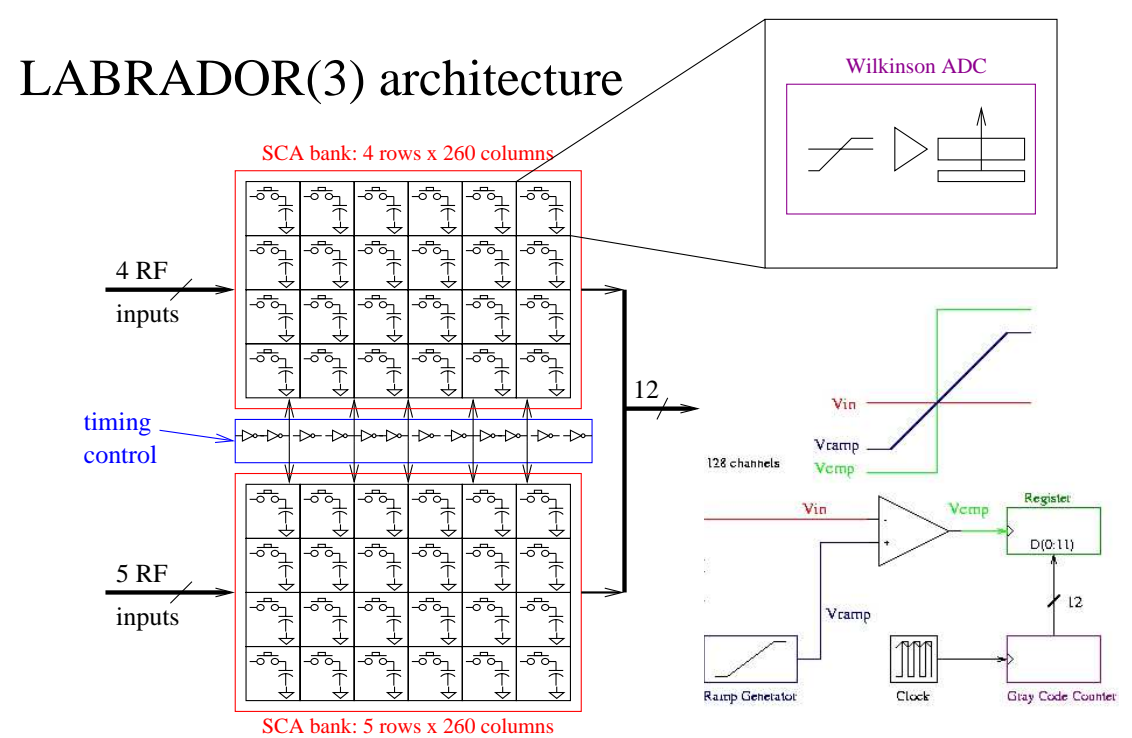

Fig. 5. Block diagram of the LAB3 architecture. In contrast to the LAB1/LAB2, the stored analog signal is never transferred. Instead, direct Wilkinson conversion is done within each storage cell.

Details of the required timing and offset calibrations are discussed below. In order to accomodate the additional samples, as well as provide space for a Wilkinson comparator and 12-bit latch in each pixel storage cell, the die had to increase slightly to approximately 3.2 by $2.8 \mathrm{~mm}$. Metal fill rules required covering the interesting parts of the die, making LAB3 far less photogenic than LAB1/LAB2 and thus not included. Addition of a 9th channel was done to allow insertion of a common reference clock into the data stream for each LABRADOR. This was found to be use for improving the temporal alignment of waveforms recorded by different chips.

All three generations use the same write pointer structure. This is a classical voltage-controlled inverter chain, with an odd number of stages such that a ripple continuously propages. An XOR circuit and a look-ahead signal are used to open each storage gate for the time it takes to transition from the look ahead to current locations (4-6 samples).

Despite best efforts at balancing the threshold voltage and NMOS versus PMOS L:W ratios, some amount of propagation variation is expected when the ripple edge across the array is transitioning low-to-high versus high-to-low, as shown below.
The ramping voltage for Wilkinson conversion is generated by using a current source and either an internal or external reference capacitor. In all testing shown below, an external 200pF capacitor is used. An external $(68 k \Omega)$ bias resistor sets the drive strength of the current source to approximately $20 \mu \mathrm{A}$. A common Gray-code counter is provided on chip and broadcast to all SCA cells. When the ramp threshold is crossed in a particular cell, the current count value is latched. Upon completion of ramping, all 2340 12-bit values are available for random-access readout.

\subsection{Design Evolution}

In summary, the biggest changes in going from the LAB1/LAB2 architecture to the LAB3 are

(i) direct termination at array input

(ii) Wilkinson conversion in each storage cell (no analog signal transfer)

(iii) addition of a 9th (clock reference) channel

and by these choices good performance results have been obtained, as documented below. 


\section{Test Results}

A variety of tests have been carried out to evaluate the performance of the LABRADOR series of waveform recorders. These measurements attempt to verify the degree to which the performance targets have been met, as well as characterize the system in preparation for UHF radio transient detection. Because of the superior performance of measured noise, bandwidth, linearity and digitization, results are shown the LAB3 ASIC.

\subsection{Sampling Speed}

The sampling speed dependence on an adjustable control voltage (ROVDD) is plotted in Fig. 6. Stable sampling speeds ranged from 0.02 to almost $4 \mathrm{GSa} / \mathrm{s}$ (limited by operation beyond the $2.5 \mathrm{~V}$ nominal VDD rail voltage).

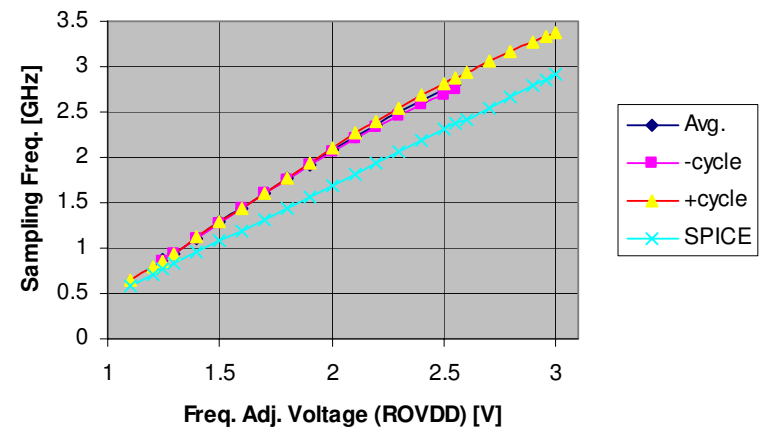

Fig. 6. Sampling rate as a function of control voltage. Both data and SPICE simulation are plotted, where a difference is observed between rising or falling edges of the ripple oscillator as described in the text.

The SPICE simulation was fairly conservative and should be considered a lower-limit, pessimized for a worst-case spread in actual CMOS fabrication parameter values. While the sampling rate is defined as the cycle average of the so-called Ripple Carry Out (RCO), which is a copy of the write pointer monitored external to LAB3, the propagation speed of the high-to-low and low-to-high are seen to be different. At a nominal $2.6 \mathrm{GSa} / \mathrm{s}$ this corresponds to about a $2 \%$ effect and is readily calibrated out by latching the RCO bit state at the time a trigger is recorded, as will be discussed later.

\subsection{Input Coupling}

Pulsing the input to the LAB3 chip with a fast risetime signal, a reflection $R=+6.8 \%$ is observed. Solving the usual expression

$\frac{Z-Z_{0}}{Z+Z_{0}}=R$

an impedance value of $Z=57 \Omega$ is determined. This is consistent with the measured $59 \Omega \mathrm{DC}$ resistance of the fabricated device, which appears to be about $20 \%$ higher than specified, though within spreads observed for silicide block in recent similar runs.

Because the signal of interest is an RF signal, a standard DC linearity scan performance is less important than evaluation with a realistic impulsive signal. Therefore, to determine the input coupling, linearity and cross-talk performance, an RF impulse was used as shown in Fig. 7. Most of the signal power of interest is in the steep high-to-low transition.

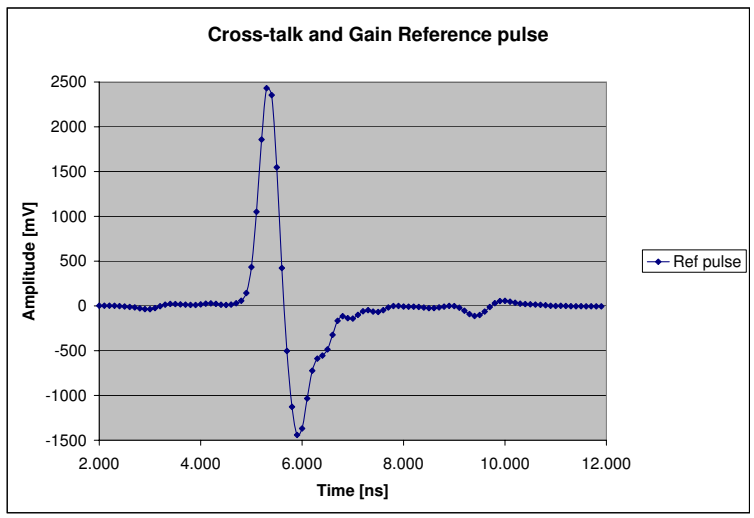

Fig. 7. Time-domain signal of the RF pulse used to evaluate input coupling, linearity and crosstalk, as recorded with a $3 \mathrm{GHz}$ bandwidth oscilloscope.

A $3 \mathrm{GHz}$ analog bandwidth oscillocope was used to record this reference signal. However the signal from the pulse generator itself was not flat in the frequency domain. Moreover this reference pulse has been bandwidth limited between 200$1200 \mathrm{MHz}$, to match the frequency range of the ANITA instrument signal chain, in which these measurements have been performed. 
Because determination of the analog bandwidth of the LAB3 device requires removing the intrinsic frequency of the RF pulse itself, its FFT has been measured and is displayed as the blue curve in Fig. 8. In red in this upper plot is the recorded LAB3 response.
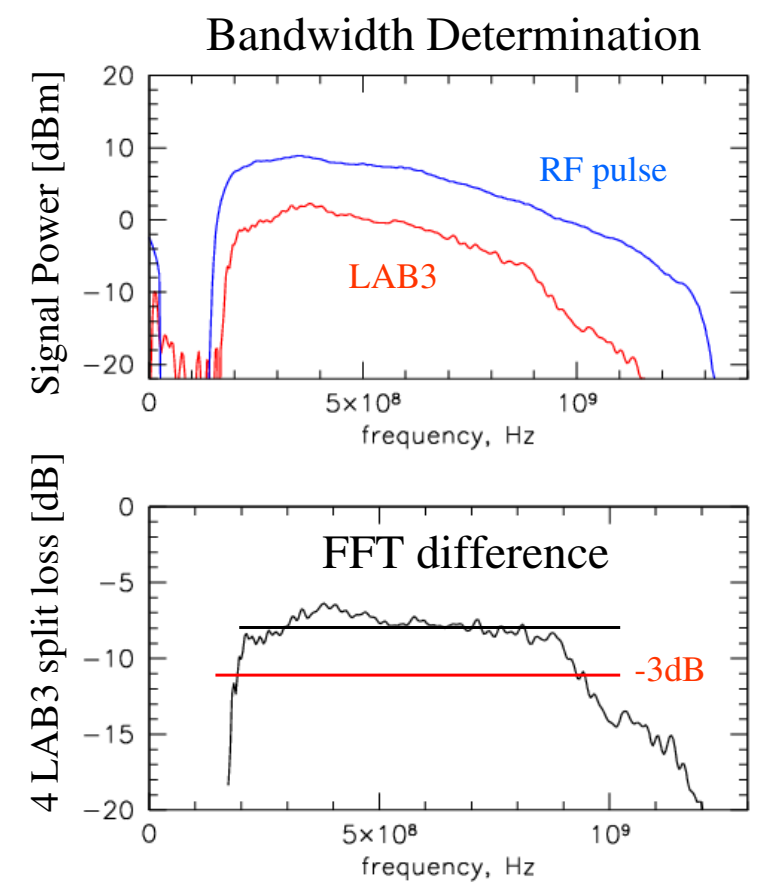

Fig. 8. Determination of the LAB3 ASIC analog bandwidth in a test board configuration with a four-way split of the RF signal. In top (blue) RF reference pulse and (red) LAB3 FFT. At bottom is the difference, where for perfect coupling a $-6 \mathrm{~dB}$ loss would be expected.

Taking the difference of these two curves, the analog response versus frequency is determined and shown in the bottom plot of Fig. 8. At the left edges of the curves the impact of the $200 \mathrm{MHz}$ high-pass band definition filters are seen. Of note is peaking of the signal in the $300-400 \mathrm{MHz}$ range, an effect seen in earlier testing. Taking the $-3 \mathrm{~dB}$ point as the line shown, the roll-off frequency is just over $900 \mathrm{MHz}$, though signal power is still available out to $1200 \mathrm{MHz}$. Four LAB3 are being tested in parallel and thus an ideal loss would be $-6 \mathrm{~dB}$, indicating some amount of loss in the RF signal chain and coupling into the chip. Earlier tests on a dedicated, single LAB3 board, without band definition filters (e.g. $1200 \mathrm{MHz}$ low pass) indicated somewhat better higher frequency response and some of this loss may be due to components on the ANITA flight digitizer (SURF[2]) board used for evaluation. Therefore this curve may be considered a conservative lower bound on the analog bandwidth.

We note that the peaking observed is also present in the case of gaussian noise, though the peak of the distribution is a function of the input biasing network. This is likely due to resonant L-C response in the input front end and seems coupled to the cross-talk observed below.

\subsection{Linearity}

A determination of the linearity of the digitizing system has been made by varying the RF signal amplitude as displayed in Fig. 9.

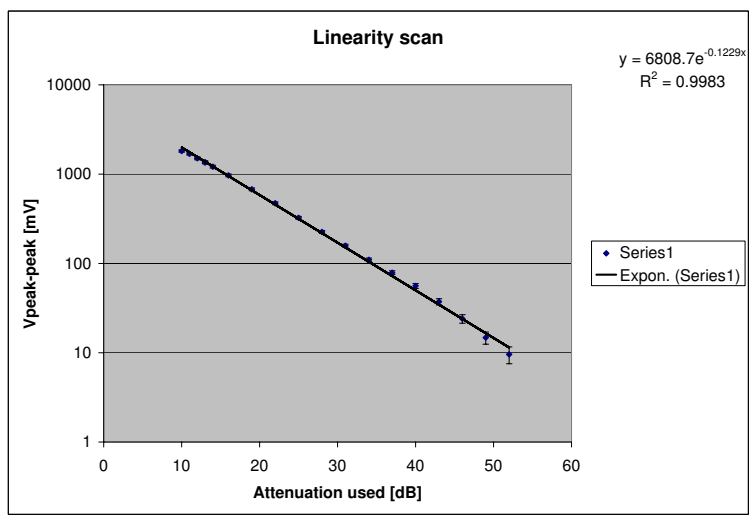

Fig. 9. Linearity determined by attenuating an RF pulse as described in the text.

Since power attenuators are used, the response is characterized in $\mathrm{dB}$ and a linear fit is observed on a logarithmic plot. Good linearity is seen with just a hint of saturation at large signal amplitudes and some non-linearity at small signal amplitude due to the coaddition of board-level noise. Any nonlinearity observed is likely due to non-linearities in the ramp generation circuit or comparator bias setting. Over a span of $40 \mathrm{~dB}$ in dynamic range, the LAB3 output tracks input to within statistical measurement errors. 


\subsection{Crosstalk}

By inserting the signal successively into the LAB3 channels, a cross-talk correlation plot was constructed as shown in Fig. 10.
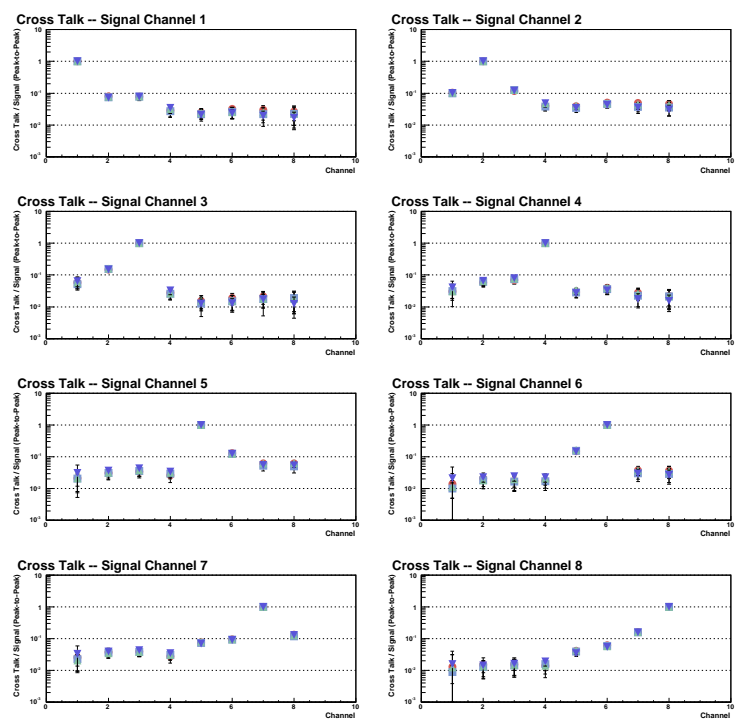

Fig. 10. Measured crosstalk for each channel as a function of channel into which signal is injected. For signal in self-channel, the amplitude is unity. Note that these values are overestimated, as described in the text.

These values shown are determined by searching for a peak around the time of the input signal. Due to noise, statistically a few percent peak is measured even for the case of no cross-talk. Therefore the values shown are overestimated. For RF applications, even a $10 \%$ voltage crosstalk is only $1 \%$ in power.

Nevertheless, for other applications it is important to understand the source of this effect. A hint to the origin of this crosstalk may be seen in Fig. 11.

Similar temporal and frequency dependence to the cross-talk can be reproduced in SPICE simulations, though the solutions are not unique. That is, the amplitude and phase information can be mimicked by tuning the voltage source output inductance of the pedestal network or with respect to bond-wire inductance stray coupling. Based on these results, a channel-dependent phase-lag to the cross-talk was predicted and subsequently verified qualitatively, as shown in Fig. 12.

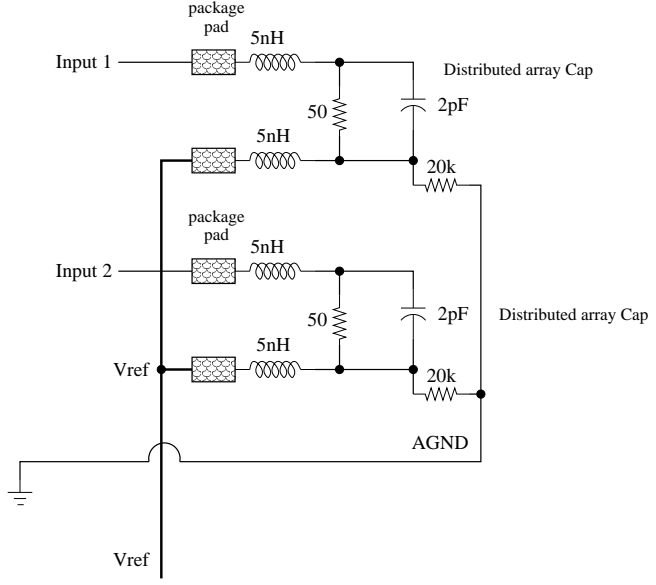

Fig. 11. Schematic representation of the input bias circuit.
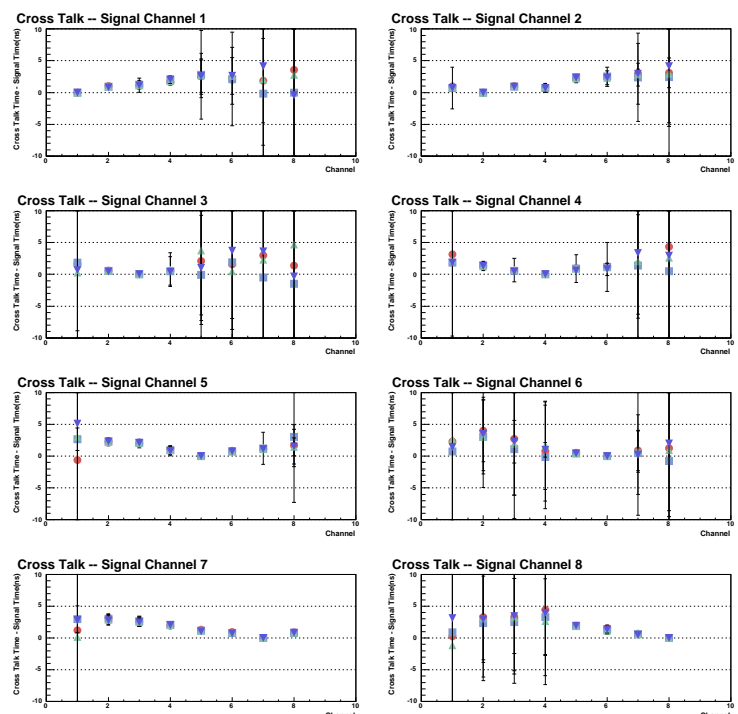

Fig. 12. Phase lag of the measured crosstalk. For channels separated by the timing control section of the chip both the amplitude and phase are less well constrained.

In addition, a small component of direct radiative coupling between the on-chip striplines cannot be ruled out, though was difficult to model (metalic heat sinks would need to be taken properly into account in the 3D EM simulations). All results indicate that better packaging (lower inductance) and stripline shielding would help improve the observed effects. 


\section{Required Calibrations and Stability}

In order to obtain the test results shown, a number of calibrations are needed. In the process of applying these, much improved resolution is obtained. Temperature dependence and timing precision limits are considered.

\subsection{Gain and Pedestal Calibration}

For the measurements shown, the gain has been adjusted to approximately $1 \mathrm{mV}$ /least count. A comprehensive pedestal histogram of all SCA storage channels (excluding channel 9) on the 36 LAB3 flown on ANITA is summarized in Fig. 13.

\section{Pedestal Distribution}

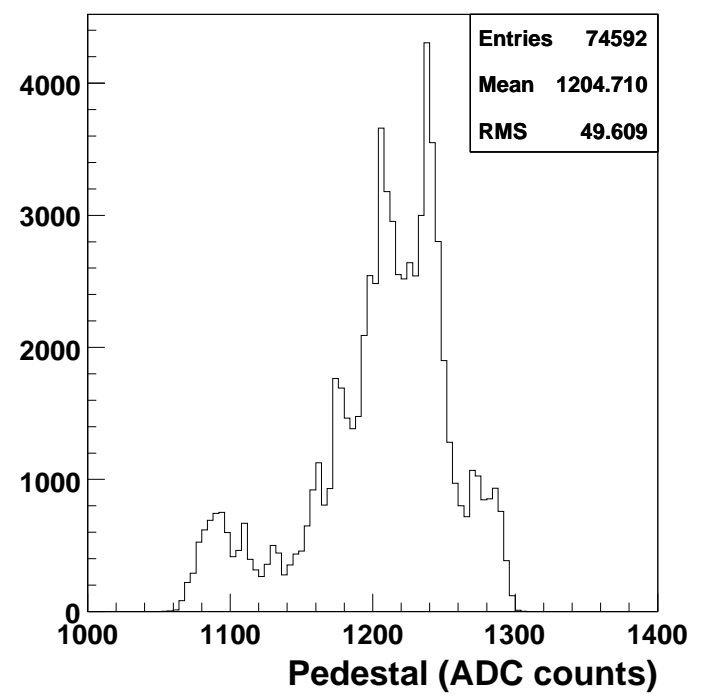

Fig. 13. A summary of the pedestal values (in $\mathrm{mV}$ ) for all SCAs of 36 production LAB3 tested.

Channel 9 is excluded since it has a different voltage offset value due to the clock input biasing. The spread seen is a combination of 36 pedestal voltage differences, SCA-SCA variations, and Wilkinson ramp slope and starting voltage offsets. Also the gain of one LAB3 (values clustered around 1100) had an anamolously low gain. Overall the RMS of this distibution is just over $4 \%$.

\subsection{Timing Calibrations}

In order to obtain the best possible timing resolution, a number of calibrations, due to the method in which the sampling is implemented, must be considered. As mentioned earlier, the write pointer is monitored using a copy of the signal called RCO. Since the sampling is done in so-called Common Stop mode, it is continuous until a trigger condition is formed. Thus all samples have already been recorded by the time a trigger is acted upon. In order for sampling to be continuous it is necessary for the write pointer to wrap around from the end of the array to the beginning, as illustrated in Fig. 14.

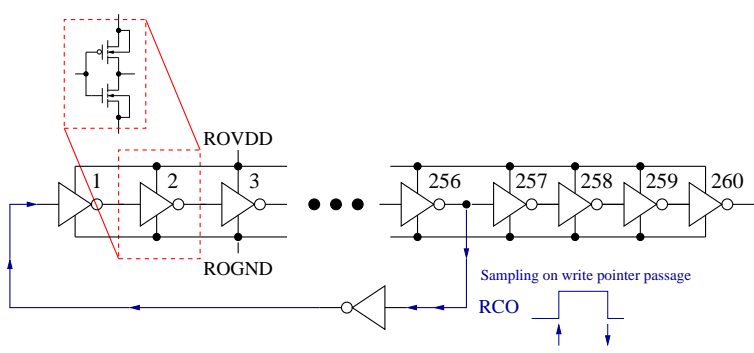

Fig. 14. Write pointer wrap around. While the write pointer returns to position 0 of the array, additional tail samples are taken in order avoid a gap in the sampling record.

Four additional "tail" samples are provided to permit samples to be recorded during the time in which the write pointer is returning to the beginning of the array. Even though the physical distance is only 20-30ps at the speed of light, the need to go through an additional inverting stage (to form ring oscillator) and the capacitance associated with the long signal line back to the beginning of the array limit the speed of write pointer return.

Also mentioned earlier, the write pointer speed of propagation across the array is a function of the transition direction. Likewise the delay time of write pointer return is also RCO phase dependent. The most general case of these calibration constants is illustrated in Fig. 15. From the measured $\mathrm{RCO}$ frequency $\left(f_{\mathrm{RCO}}\right)$, the sampling frequency is determined as

$f_{\text {sampling }}=2 \times 256 \times f_{\mathrm{RCO}}$. 


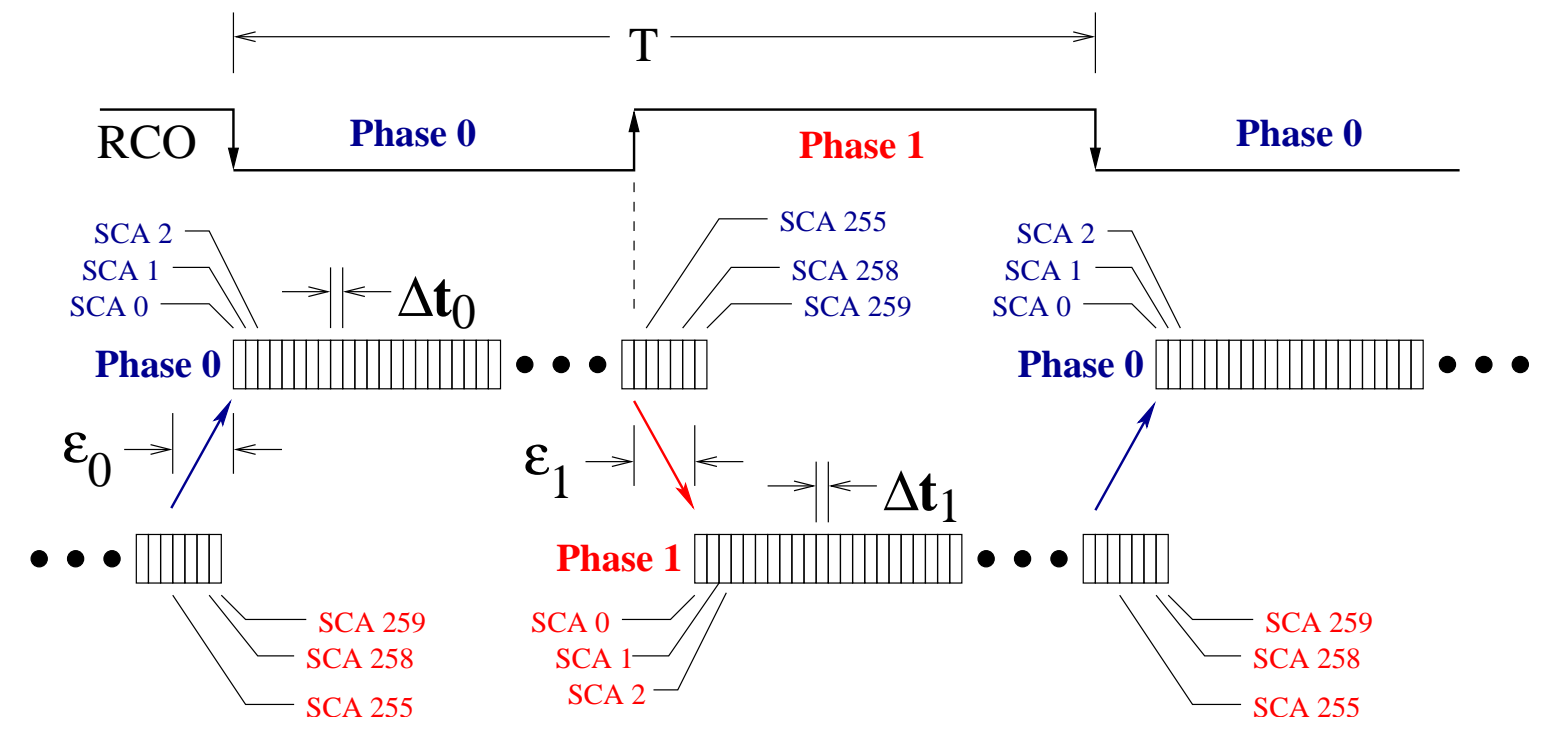

Fig. 15. Definition of the most general LAB3 sample timing relationships and constants. Determination of their values is described in the text.

Expressing $f_{\mathrm{RCO}}$ in terms of its period $T$, half period $T_{0}$ corresponds to $\mathrm{RCO}$ phase 0 and half period $T_{1}$ corresponds to RCO phase 1 , or

$f_{\text {sampling }}=512 \times\left(T_{0}+T_{1}\right)^{-1}$

in which case the time step of an individual sample is expressed as

$\Delta t=\frac{T}{512}$.

In general, as mentioned, the half periods $T_{0}$ and $T_{1}$ are not half the period $T$ :

$T_{0} \neq T_{1} \neq \frac{T}{2}$

which means that the average individual time steps in phase $0\left(\Delta t_{0}\right)$ are different from those in phase $1\left(\Delta t_{1}\right)$. Likewise the delay time of the write point propagation for RCO $0 \rightarrow 1\left(\epsilon_{1}\right)$ and for RCO $1 \rightarrow 0$ are in general different and related to the difference between average $\Delta t_{0}$ and $\Delta t_{1}$. Finally, due to transistor threshold dispersion, the actual widths of each of the time bins $\left(\Delta t_{0,1}^{0.259}\right)$ can be slightly different.

Using a known periodic input signal, it is possible to generate calibration values for all of these parameters. An example of determination of the relative average $\Delta t_{0}$ and $\Delta t_{1}$ is shown in Fig. 16.
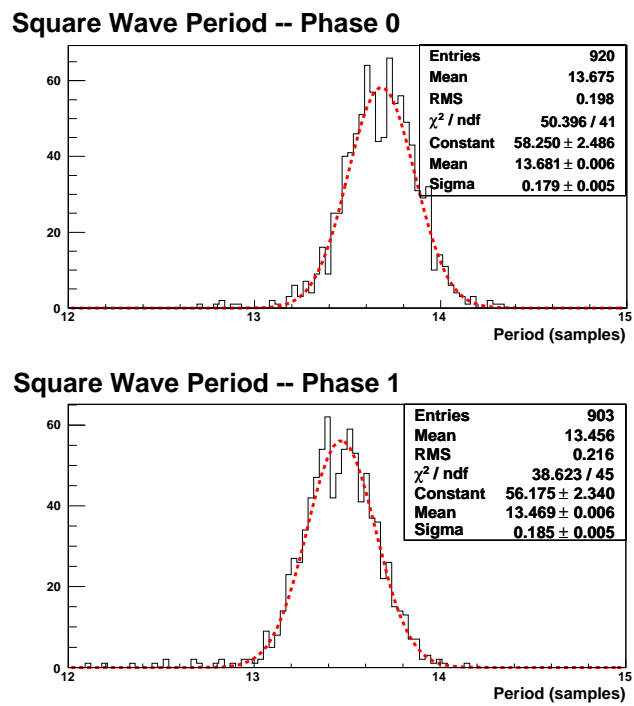

Fig. 16. Measurement of the write pointer propagation (sampling speed) difference for the RCO $=0$ (top) and $\mathrm{RCO}=1$ (bottom) phases for a $200 \mathrm{MHz}$ reference clock.

In each case the variable parameter is tuned until the spread or offset in the determined period is minimized. Because the period is well determined, the procedure is very efficient and requires a relatively small amount of calibration data. 
Similarly, the write pointer wrap around delays, $\epsilon_{0}$ and $\epsilon_{1}$, may be determined by constraining the measured period to be consistent across the write pointer wrap around. An example is shown in Fig. 17.
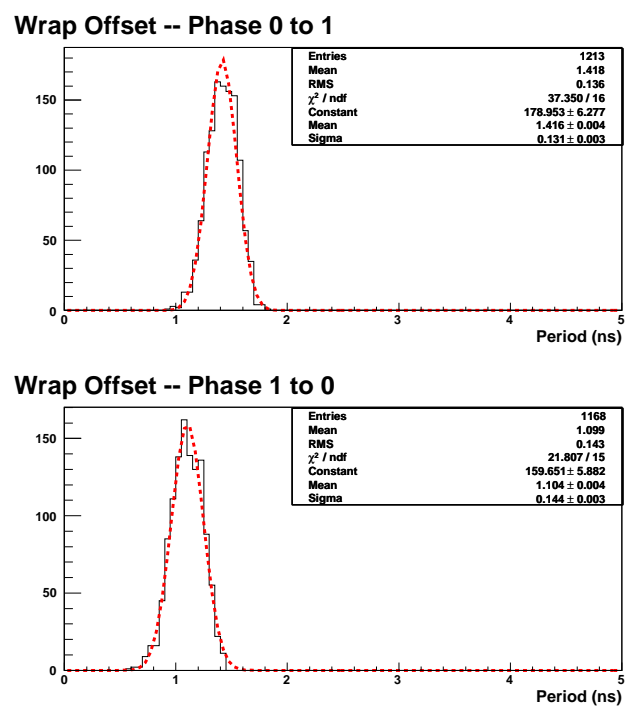

Fig. 17. Extraction of the wrap timing offsets $\left(\epsilon_{0}\right.$ and $\left.\epsilon_{1}\right)$ for a given LAB3.

To a certain extent these calibration steps must be bootstrapped. For example, correctly minimizing the error on these $\epsilon$ parameters requires that the average time steps in each of the RCO phases be correctly determined. A subtlety here is that the $\mathrm{RCO}$ phase is recorded at the time a trigger signal (hold) is issued. Because the RCO latching in the data is not completely synchronous, there is in general a delay between the measured value of RCO and its actual value. This ambiguity is resolved by assigning a phase delay between the measured RCO that depends upon the address at which the hold was issued, the so-called "HitBus" value. The value of this delay is tuned in the data until the width of the measured period is again minimized.

Finally, using a high frequency clock it is possible to constrain the average half period and assign its average value to the $\Delta t_{0,1}^{0.259}$ bin in which the positive/negative lobe peaks. Using this prescription the distribution histogrammed in Fig. 18 is obtained.

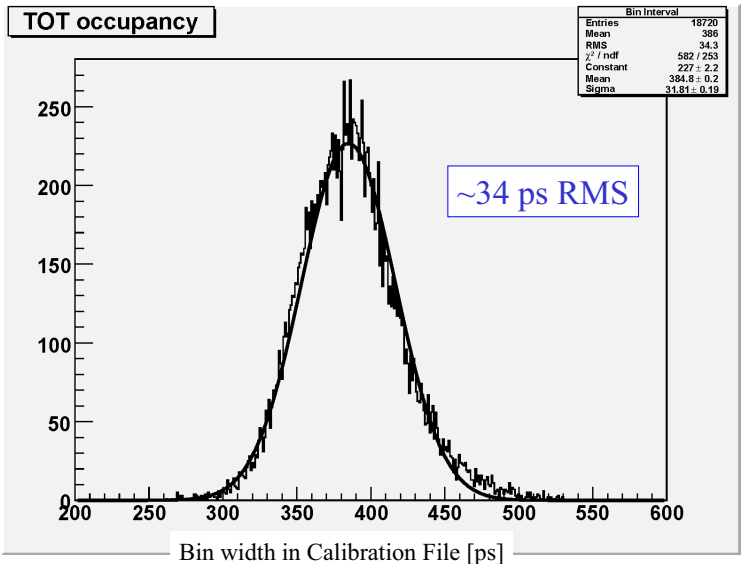

Fig. 18. Summary distribution of the calibrated individual time bin widths for all SCAs in 36 LAB3 ASICs.

\subsection{Time Resolution Limitations}

Applying these timing corrections to the data leads to an improvement in the time resolution of signals in the data. The precise improvement depends upon the signal distance within the window (cumulative error) and method for correlating signal shapes to extract a timing feature for comparison.

To understand the intrisic performance limits and the significance of the bin-by-bin correction, a simple Monte Carlo study was performed to determine the extent to which the technique used to extract the observed timings would lead to the observed distribution. Introducing a completely random scatter (uniform distribution) of $15 \%$ to the nominal $386 \mathrm{ps}$ bin width, $600 \mathrm{MHz}$ sine $\mathrm{MC}$ was then synthesized and the algorithm applied. A value of $15 \%$ was determined empirically to provide a good representation of the observations in data. Due to irreducible errors in the specific implementation of this zero-crossing technique, application of these constants improves the timing resolution to about 28ps, as shown in Fig. 19. This has improved the resolution by about 20ps in quadrature, though perhaps there is still room for improvement. Since two edges are used to determine this time interval, the single edge measurement is about $28 \mathrm{ps} / \sqrt{2}$ or about $20 \mathrm{ps}$ and probably is a limit with the current LAB3. 


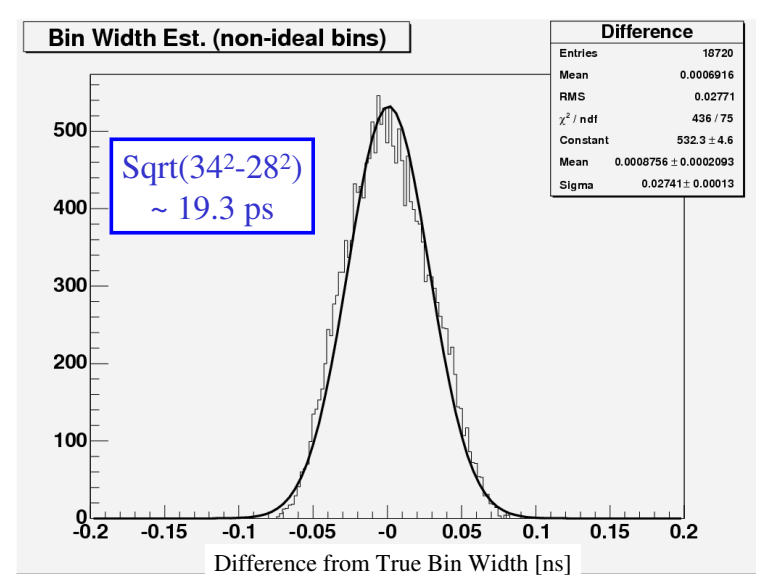

Fig. 19. Monte carlo.

These determined parameters appear to be stable in time and only depend upon thermal effects, and described next.

\subsection{Temperature Dependence}

The voltage controlled oscillator for the write pointer is fundamentally temperature dependent. During operation of LAB1 an external delay locking loop circuit was used to adjust ROVDD to compensate. However this circuit suffered from large phase noise as well as a nasty habit of locking onto a frequency subharmonic at power-on. Therefore, with the addition of a dedicated timing channel needed to precisely align multiple LAB3 waveforms offline - ROVDD was fixed and timebase correction is implemented by fitting to the period of the reference clock.

The temperature dependence of the sampled frequency is shown in Fig. 20. Good agreement is seen with SPICE simulations of the temperature dependence, once an operating reference point is set. This fine tuning is needed to correct for the overly pessimistic parasitic capacitance estimate used earlier in simulating the ripple oscillator frequency. Using the reference clock signal on channel 9, this temperature dependence of the $\mathrm{VCO}$ is corrected in the offline analysis. A fit to this dependence gives a change of approximately $55 \mathrm{ps} /{ }^{\circ} \mathrm{C}$ over the $30 \mathrm{~ns}$ period of the $33 \mathrm{MHz}$ reference clock, or about $0.2 \% /{ }^{\circ} \mathrm{C}$.

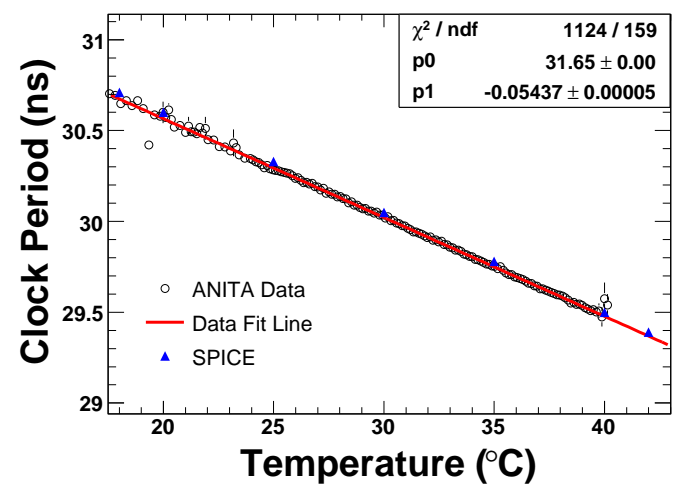

Fig. 20. Measured and SPICE simulated temperature dependence of the LAB3 sampling period.

In contrast, the pedestals are a very weak function of temperature. In Fig. 21 is displayed the difference in pedestal values taken after an ambient temperature change of approximately $17^{\circ} \mathrm{C}$.

\section{Pedestal Stability}

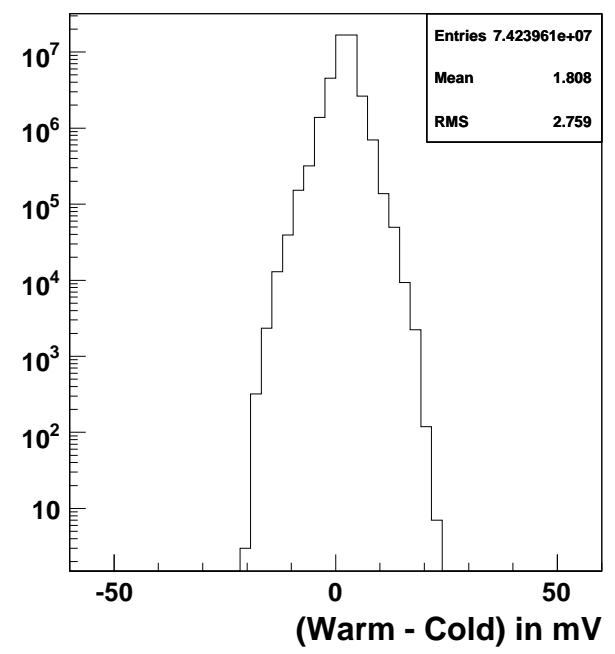

Fig. 21. Difference in pedestal between dedicated pedestal runs taken 30 hours apart, at a difference in ambient temperature of 17 degrees Celcius.

Taking this difference, an estimate of the pedestal temperature dependence is

$\mathrm{PED}_{\mathrm{avg}}=+0.052 \cdot \frac{\mathrm{ADC} \text { counts }}{{ }^{\circ} \mathrm{C}}$ 
For reference, and to illustrate the typical chiplevel noise, an example noise run is shown in Fig. 22. Representative noise values are about $1.3 m V_{r m s}$, though there is some non-gaussian behavior in the combined distribution of 2.2 million samples from 9 separate RF channels.

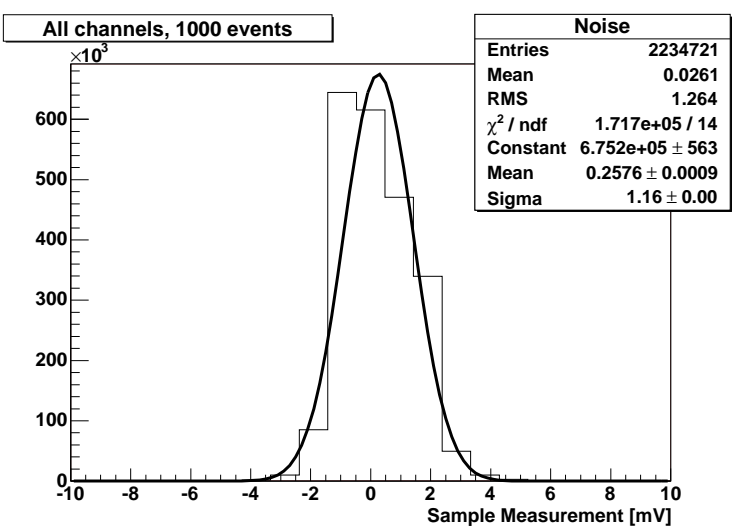

Fig. 22. Sample LAB3 1k event noise run, with all 9 channels combined into a single distribution.

\subsection{Interleaved Sampling}

Right at Nyquist sampling of UHF RF sine wave signals visually appears undersampled to most observers. This is due to expectations from seeing smooth curves generated by $20+\mathrm{GHz}$ offset interleaved sampling of a repetitive waveform, provided by most digital signal oscilloscopes. By providing precise external delays it is possible to enhance the sampling speed and provide oversampling with the LAB3 chip. Interleaving of 8 inputs, running at $2.5 \mathrm{GSa} / \mathrm{s}$ each has been done to provide singleshot recording of a $400 \mathrm{MHz}$ sine wave signal at $20 \mathrm{GSa} / \mathrm{s}$, as shown in Fig. 23. Each color represents the samples recorded by a single channel.

While there is some scatter due to the delays not being perfectly tuned, this indicates that there is still more performance to be gained by increasing the analog bandwidth yet further and implementing such interleaving. For low power and very high sampling rate applications, where signals may not be repetitive, this technique may be useful. This and other improvements are considered next.

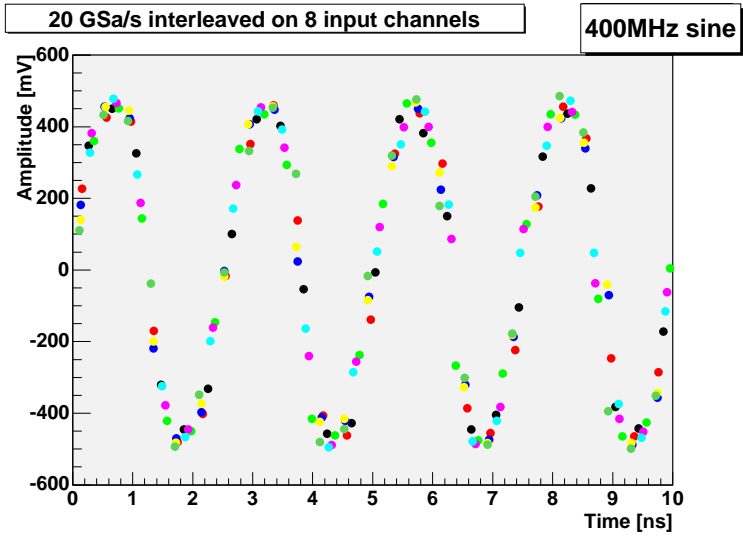

Fig. 23. Example of $20 \mathrm{GSa} / \mathrm{s}$ interleaved, single-shot waveform recording of a $400 \mathrm{MHz}$ sine wave signal on 8 LAB3 input channels, each plotted with a different color.

\section{Future Directions}

Beyond increasing analog bandwidth, to fully exploit the enhanced sampling speed of deep submicron processes, there is a desire to increase sampling depth. This is being explored in a followon device designated the Buffered LABRADOR (BLAB) ASIC. While the sampling speed increases below $0.25 \mu \mathrm{m}$, loss of dynamic range due to reduced rail voltages and increased leakage current may preclude going to smaller feature sizes.

\section{Applications}

During December 2006 to January 2007, 36 LAB3 ASICs flew successfully at 120,000 feet for 35 days around the Antarctic continent. Some of the test results shown above are from this data set. During this same period, test deployments for an in-ice radio detector, using this device, were made at the south pole in conjunction with the IceCube array [16]. Recently, these ASICs were evaluated in a collidering beam environment for upgrade of the Belle Time-Of-Flight readout [17], and a variant for operation at a Super B-factory [18] is being developed for high timing precision, single photon recording [19]. For these future applications, a deeper sampling depth is highly desirable and such a device is currently being prototyped. 


\section{Summary}

A Switched Capacitor Array (SCA) device has been developed in a $0.25 \mu \mathrm{m}$ CMOS process with a $3 \mathrm{~dB}$ analog bandwidth of almost a Giga-Hertz, capable of being sampled at many GSa/s, or well above Nyquist minimum. Sampling is performed at low power and the entire array of 9 channels by 260 samples can be digitized to 12-bits of resolution and read out within $50 \mu \mathrm{s}$. With calibration excellent time and sample voltage resolution have been obtained over a large range of temperature and sampling speeds.

\section{Acknowledgements}

This work was supported by the National Aeronautics and Space Administration (ROSS Program), the Department of Energy (HEP Division) University of Hawaii base program support as well as support from the Advanced Detector Research program.

\section{References}

[1] P.W. Gorham et al. (ANITA Collaboration), Submitted to Phys. Rev. Lett., hep-ex/0611008 SLAC-PUB-12286.

[2] G.S. Varner et al. (ANITA Collaboration), Detection of Ultra High Energy Neutrinos via Coherent Radio Emission, Proceedings of International Symposium on Detector Development for Particle, Astroparticle and Synchrotron Radiation Experiments (SNIC 2006) pp 0046, SLAC-PUB-11872.

[3] S. Kleinfelder, IEEE Trans. Nucl. Sci.35 (1988) 151; IEEE Trans. Nucl. Sci.37 (1990) 1230.

[4] K.L. Lee et al., IEEE Trans. Nucl. Sci.38: 344-347, 1991.

[5] G.M. Haller and B.A. Wooley, IEEE J. Solid State Circuits 29 (1994) 500; IEEE Trans. Nucl. Sci. 41 (1994) 1203.

[6] S. Kleinfelder, IEEE Trans. Nucl. Sci. 50 (2003) 955.

[7] C. Brönnimann, R. Horisberger and R. Schnyder, Nucl. Instr. Meth. A420 (1999) 264.

[8] S. Ritt, Nucl. Instr. Meth. A518 (2004) 470.
[9] E. Delagnes et al., Nucl. Instr. Meth. A567 (2006) 21.

[10] S. Panebianco et al., Nucl. Instr. Meth. A434 (1999) 424 .

[11] G.S. Varner et al., Monolithic Multi-channel GSa/s Transient Waveform Recorder for Measuring Radio Emissions from High Energy Particle Cascades, Proc. SPIE Int.Soc.Opt.Eng. 4858 (2003) 31.

[12] G. Varner et al., Nucl. Instr. Meth. A541 (2005) 166; Nucl. Instr. Meth. A565 (2006) 126.

[13] P. Allen and E. Sanchez-Sinencio, Switched Capacitor Circuits, Van Nostrand Reinhold, 1984.

[14] R. Unbehauen and A. Cichocki, MOS SwitchedCapacitor and Continuous-Time Integrated Circuits and Systems, Springer-Verlag, 1989.

[15] R. Gregorian and G. Temes, Analog MOS Integrated Circuits for Signal Processing, John Wiley \& Sons, Inc., 1986.

[16] H. Landsman, A. Karle et al. (IceCube Collaboration) and G. Varner and L. Ruckman, to be published International Cosmic Ray Conference 2007 proceedings.

[17] J. Rorie and G. Varner, Signal Timing and Readout $(S T a R)$ pipelined upgrade for the Belle TOF System, to appear Proceedings of the 2007 IEEE Nuclear Science Symposium.

[18] S. Hashimoto (ed.) et al., KEK-Report-2004-4 (2004).

[19] L. Ruckman and G. Varner, Photodetector Readout Monolithic with Precision Timing, to appear Proceedings of the 2007 IEEE Nuclear Science Symposium. 